\title{
Fractal Charge Carrier Kinetics in $\mathrm{TiO}_{2}$
}

\author{
Fabian Sieland, $*^{\dagger} \dagger$ Jenny Schneider, ${ }^{*}{ }^{\dagger}$ and Detlef W. Bahnemann ${ }^{\dagger,+}$ \\ ${ }^{\dagger}$ Institute of Technical Chemistry, Leibniz University Hannover, Callinstrasse 5, 30167 Hannover, Germany \\ ${ }^{\ddagger}$ Laboratory "Photoactive Nanocomposite Materials", Saint-Petersburg State University, Ulyanovskaya Str. 1, Peterhof, Saint \\ Petersburg, 198504, Russia
}

\section{Supporting Information}

ABSTRACT: Charge carrier recombination kinetics of $\mathrm{TiO}_{2}$ powder samples were analyzed in the time domain ranging from $50 \mathrm{~ns}$ to $1 \mathrm{~ms}$. The transient reflectance signals of the charge carriers observed by laser flash photolysis spectroscopy do not fit to simple second order kinetics as expected for the recombination of trapped electrons and holes. The deviation from second order reaction dynamics could rather be explained by the segregation of charge carriers and the fractal dimension of the semiconductor agglomerates. According to the fractal reaction kinetics, the time dependent rate coefficient $k_{f}\left(k_{f}=k_{2, f} t^{-h}\right)$ has been employed instead of the second order rate constant $k_{2}$, where the fractal parameter $\mathrm{h}$ describes the dimension of the system. This model could successfully be used to describe charge carrier signals in all observed time domains.

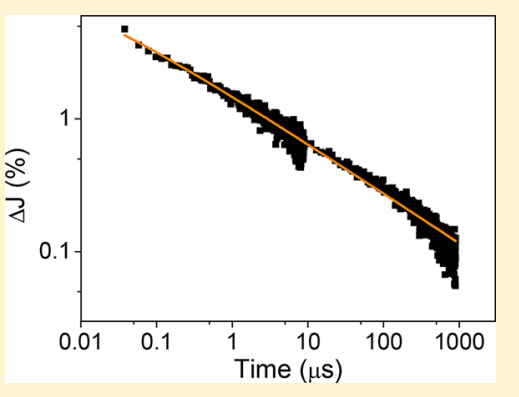
Moreover, the model was compared with the concept developed by Shuttle et al., which proposes that the charge carrier signals decay following a power-law. The benefits of the fractal model proposed here include the possibility to describe and analyze influences of the morphology on the fractal parameter $h$ and its applicability over a broad range of time domains and excitation energies.

\section{INTRODUCTION}

The lifetime of photogenerated charge carriers represents one of the most important parameters influencing the application of semiconductors in photocatalytic, photoelectrochemical, and photovoltaic processes. ${ }^{1-4}$ Only with sufficiently long charge carrier lifetimes and appropriate charge carrier diffusion lengths will the excited charge carriers be able to reach the surface or the back contact of an electrode to drive a reaction. ${ }^{1}$ The short charge carrier lifetime in hematite, for instance, is responsible for its rather bad photocatalytic performance in comparison to titanium dioxide. ${ }^{5,6}$ Subsequently, it is of utmost importance to determine the charge carrier dynamics, which can be used to evaluate their lifetime. In the last decades different techniques were employed to analyze the charge carrier kinetics, for example, photoluminescence, ${ }^{7,8}$ transient absorption spectroscopy, ${ }^{9,10}$ and time-resolved microwave conductivity. ${ }^{11-13}$ Charge carrier signals have been analyzed in time domains ranging from femtoseconds ${ }^{10,14}$ to seconds ${ }^{15}$ and, mainly, characterization has been performed by direct comparison of the signals under varied experimental conditions. ${ }^{10}$ Typically, nitrogen and argon atmospheres were applied as reference conditions and methanol and oxygen were used as hole and electron scavengers, respectively. ${ }^{4,9,10,16,17}$ However, a detailed kinetic analysis of the decaying charge carrier signal is required for an in-depth understanding of the underlying processes.

In most experimental studies charge carrier signals in $\mathrm{TiO}_{2}$ were fitted mainly with monoexponential, ${ }^{14}$ double-exponential, ${ }^{18}$ and second order ${ }^{10,19-21}$ decay functions. Katoh et al. provided detailed kinetic analysis, related to the second order processes, unfortunately focusing only on signals in the picoseconds to nanosecond regime. ${ }^{22}$ In 1990, Schindler and
Kunst reported transient photoconductivity in $\mathrm{TiO}_{2}$ decays following a power-law. ${ }^{23}$ Tachiya and Mozumder reported already in 1975 that the kinetics of geminate ion recombination by electron tunneling follows a power law for an exponential distribution of distances between the reactants. ${ }^{24}$ Further, Barzykin and Tachiya related the power law decay kinetics of electrons in dye sensitized $\mathrm{TiO}_{2}$ particles to a multiple-trapping model. $^{25}$ On the basis of these results and an exponential distribution of trapped states in the bandgap ${ }^{26}$ Shuttle et al. ${ }^{27}$ developed a model leading to power-law decays of the transient absorption signals of the charge carriers, with the effective charge carrier mobility depending on the charge density. ${ }^{27,28}$ However, this model does not include charge transfer between different particles and assumes immobile hole species. ${ }^{25}$ Furthermore, it includes the simplification that long times after the generation the recombination mechanism of the charge carriers is influenced solely by the trap energy distribution. ${ }^{25}$ At shorter times, on the other hand, the recombination is also governed by structural parameters (e.g., the random walk mechanism) ${ }^{25}$ Unfortunately, the authors do not provide information concerning the time limitation, that is, after which time interval between charge carrier generation and observation their model can be applied. Hence, the power-law model has predominantly been applied for charge carrier signals from microseconds to seconds following their generation, provided that very low excitation energies are used. ${ }^{6,28,29}$ Consequently, the fitting of entire transient photoluminescence

Received: July 18, 2017

Revised: September 27, 2017

Published: October 4, 2017 
signals observed by Wang et al. required a combination of the power-law model with an exponential decay curve. ${ }^{30}$ To the best of our knowledge, there is currently no model available for the analysis of charge carrier dynamics in both short and long time scales and at low and high excitation energies. While Barzykin and Tachiya have recently developed a stochastic model for the recombination of excitons initially formed in semiconductor nanoparticles, the focus of the present paper is the kinetic analysis of the recombination of trapped charge carriers in these systems. ${ }^{31}$

As an alternative, the present work proposes a fitting model based on fractal geometry. Fractals are mathematical objects possessing effective "fractal" dimensions that are not necessarily equal to 1, 2, or 3 dimensions of the Euclidean space. This concept of fractals was published by Mandelbrot in 1983 and discussed for heterogeneous surfaces by Pfeifer and Avnir. ${ }^{32,33}$ In short, bimolecular chemical reactions such as the charge carrier recombination occurring in heterogeneous photocatalysts exhibit different kinetic behaviors as compared with similar reactions in solution. The main reason for this difference is the geometrical diffusion limitation resulting in the segregation of the charge carriers. ${ }^{34}$ In other words, the reaction takes place on a structured two-dimensional surface rather than in a free three-dimensional space. The active species can thus not simply diffuse into the liquid/gaseous surrounding or into the solid bulk of the semiconductor particle to find reaction partners. On nanostructured surfaces or in pores this geometrical diffusion limitation can even lead to apparent onedimensional reaction kinetics. ${ }^{35}$ These influences of fractal geometries on the reaction kinetics have been discussed in detail by Kopelman in $1988 .^{35}$

Interestingly, in the past decade fractal reaction dynamics were used to describe the decay of trapped electrons (in the form of $\left.\mathrm{O}_{2}{ }^{--}\right),{ }^{36}$ the dyhydrogenation of methanol, ${ }^{37,38}$ the photodesorption of $\mathrm{O}_{2}{ }^{39}$ and the dielectric response ${ }^{40}$ of $\mathrm{TiO}_{2}$. However, fractal models are rarely applied to analyze charge carrier dynamics in general. A positive counterexample is the work of Grela and Colussi, who used stochastic calculations in $2 \mathrm{D}$ lattices to model the recombination of photogenerated electrons and holes. ${ }^{41}$ They reported that for colloidal $\mathrm{TiO}_{2}$ particles this bimolecular recombination cannot be described by simple second-order kinetics. The authors observed an exponential decay of the initial exciton signal. The subsequently formed trapped charge carriers, on the other hand, recombine with second-order rate coefficients asymptotically approaching a $t^{-1 / 2}$ dependence. Furthermore, an influence of the fractal dimensions on the charge carrier recombination kinetics was noted and analyzed in detail. The thus derived second-order rate constants were found to depend on both the excitation energy and the chosen time window and, hence, are hardly applicable for a correct description and comparison of the charge carrier kinetics. Nevertheless, second order kinetic fits including a rather arbitrary chosen baseline are frequently used to analyze the charge carrier recombination due to the unavailability of simple alternatives. ${ }^{10}$ The stochastic model of Grela and Colussi was shown to successfully fit transient absorption signals of the charge carriers obtained employing $\mathrm{TiO}_{2}$ colloids of different sizes and at different excitation energies. ${ }^{41}$ This validates the strategy of applying fractal models, relating the charge carrier recombination processes to the diffusion of at least one mobile species on the particle surface.
The model described in the present study closes the gap between the fast initial second order decay of the charge carrier signals and the long lasting power-law decays. These two models are discussed as border cases of the observed decay kinetics of the transient charge carrier signals.

\section{EXPERIMENTAL DETAILS}

Materials. $\mathrm{TiO}_{2}-1$ (Kronos1001, KRONOS International Inc.; anatase, 50-200 nm diameter), $\mathrm{TiO}_{2}-2$ (PC105, Crystal; anatase, $15-20 \mathrm{~nm}$ diameter), $\mathrm{TiO}_{2}-3$ (PC500, Crystal; anatase, 5-10 nm particle diameter). For $\mathrm{TiO}_{2}$ suspensions, ultrapure water (Milli-Q, EMD Millipore) and ethanol (Carl Roth GmbH \& Co. KG; 99,8\% with 1\% MEK) were used.

Cleaning Method of the $\mathrm{TiO}_{2}$. Prior to the laser flash photolysis experiments, the $\mathrm{TiO}_{2}$ samples were treated with an ultrasound cleaning procedure. For this $\mathrm{TiO}_{2}-1, \mathrm{TiO}_{2}-2$, or $\mathrm{TiO}_{2}-3$ was added to an aqueous ethanolic solution ( $10 \mathrm{vol} \%$ ethanol, $10 \mathrm{~g} / \mathrm{L} \mathrm{TiO}_{2}$ ) and subjected to $8 \mathrm{~min}$ ultrasound treatment $(340 \mathrm{~W} / \mathrm{L})$. The solid particles were separated via centrifugation with a maximum speed of $500 \mathrm{rpm}$ for $6 \mathrm{~h}$. To achieve a better phase separation the samples were stored overnight and the precipitates were collected. The powder samples were subsequently dried at $100{ }^{\circ} \mathrm{C}$ overnight.

Instrumentation. $\mathrm{TiO}_{2}$ powders were sonicated with a Branson 450 Digital Sonifier (102-C Converter), centrifuged with a Kendro Megafuge 1.0 and dried in an oven (Memmert $\mathrm{GmbH}+\mathrm{Co}$. KG; BE 400). Laser Flash Photolysis Spectroscopy was carried out with an Applied Photophysics LKS 80 Laser Flash Photolysis Spectrometer with an Nd:YAG laser (Quantel; Brilliant B; third harmonic, $355 \mathrm{~nm}$ ). BET surface area measurements were carried out with a Micromeritics AutoMate 23.

Transient Reflectance Spectroscopy. Prior to the experiments every sample was flushed with nitrogen gas for more than $30 \mathrm{~min}$. The measurements were performed in diffuse reflectance mode. In this method a pulsed xenon lamp (Osram XBO; $150 \mathrm{~W}$ ) is focused onto the samples and the scattered light is guided into the monochromator and detector (Hamamatsu PMT R928). Figure 1 displays a scheme of the diffuse reflectance setup. ${ }^{42}$

Signal Processing. Transient reflectance signals were detected between 700 and $400 \mathrm{~nm}$ (20 and $50 \mathrm{~nm}$ step-size). The samples were analyzed in two different time domains (10 $\mu \mathrm{s}$ and $1 \mathrm{~ms}$ ) and excited at $t=0$ with a $6 \mathrm{~ns}$ UV-laser ( 355 $\mathrm{nm}$ ). Typically, the analyzing light was pulsed to achieve a high

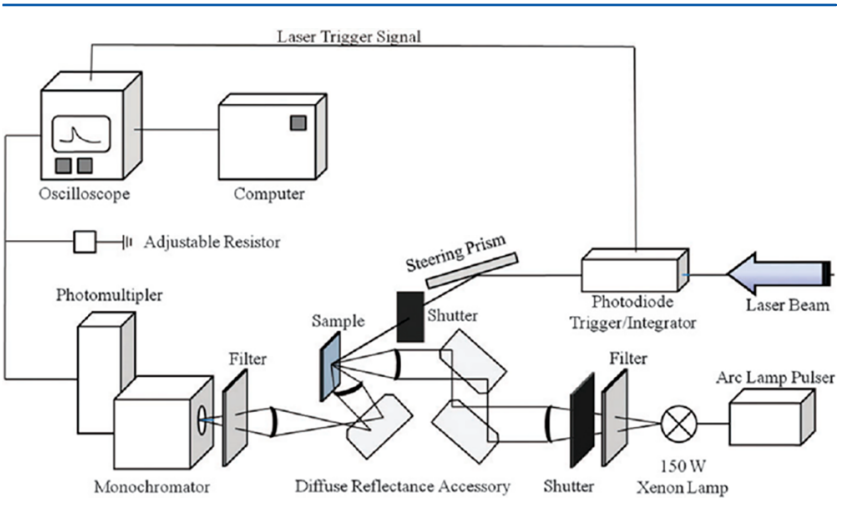

Figure 1. Scheme of the diffuse reflectance laser-flash-photolysis setup. Reprinted with permission from ref 42. Copyright 2016 PCCP Owner Societies. 
light level for the optical analysis of the samples. The pulsed light generated a stable light level for approximately $200 \mu \mathrm{s}$. Hence, the observation of the samples for long times ( $1 \mathrm{~ms}$ ) required that the lamp pulser is turned off. The reduced amount of light in the nonpulsed arc lamp operation was compensated with a higher terminal resistance and the reduced signal/noise-ratio was increased by increasing the number of averages. The experimental conditions employed for the different time scales are summarized in Table 1.

Table 1. Experimental Conditions for Transient Reflectance Spectroscopy

$\begin{array}{lll}\text { total acquired time } & 10 \mu \mathrm{s} & 1 \mathrm{~ms} \\ \text { laser frequency } & 1 \mathrm{~Hz} & 10 \mathrm{~Hz} \\ \text { terminal resistance } & 50 \Omega & 5 \mathrm{k} \Omega \\ \text { xenon lamp } & \text { pulsed } & \text { nonpulsed } \\ \text { average shots } & 12 \mathrm{shots} & 200 \mathrm{shots} \\ \text { time resolution } & 100 \mathrm{ps} / \text { point } & 100 \mathrm{~ns} / \text { point } \\ \text { laser energy } & 1 \mathrm{~mJ} / \mathrm{cm}^{2} & 2 \mathrm{~mJ} / \mathrm{cm}^{2} \\ \text { laser wavelength } & 355 \mathrm{~nm} & 355 \mathrm{~nm}\end{array}$

For every transient signal, 10000 data points were acquired, resulting in a time resolution of $100 \mathrm{ps/point} \mathrm{and} 100 \mathrm{~ns} /$ point, respectively. Generally, in the course of every measurement the employed photomultiplier detects a current depending of the amount of photons hitting the detector. The software of the instrument calculates the absorbance from the reflected light before the laser exciation $I_{0}$ and the reflected light after the laser exciation $I$ eq 1.

$$
\text { Abs }=\log \frac{I_{0}}{I}
$$

The stated correlations include the assumption that during the laser excitation the scattering properties of the sample remain constant. Figure 2 shows these absorbance raw data of $\mathrm{TiO}_{2}-2$ for $10 \mu \mathrm{s}$ as an example.

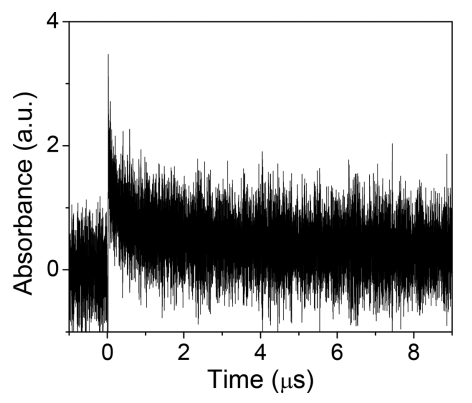

Figure 2. Transient absorbance of $\mathrm{TiO}_{2}-2$ at $500 \mathrm{~nm}$ following a $1 \mathrm{~mJ} /$ $\mathrm{cm}^{2}$ laser pulse with $355 \mathrm{~nm}$ (raw data; 10000 points; $10 \mu \mathrm{s}$ ).

For easier processing and mathematical fitting of the transient signals, the acquired set of data points was reduced to 500 points by averaging 200 reflectance values to one data point. Additionally, the optical reflectance changes $\Delta J$ of the powder samples were calculated from the absorbance values eq 2.

$$
\Delta J=1-10^{-A b s}=\frac{I_{0}-I}{I_{0}}
$$

The change in reflectance $\Delta J$ represents the fraction of the light that is absorbed by the transient species. Lin and Kan have shown in 1970 that the optical change in reflectance depends linearly on the concentration of the transient species, as long as the changes in reflectance are considerably small $(\Delta J<0.1){ }^{43}$ Figure 3 displays the processed transient data of $\mathrm{TiO}_{2}-2$ in the $10 \mu \mathrm{s}$ and the $1 \mathrm{~ms}$ time domain.
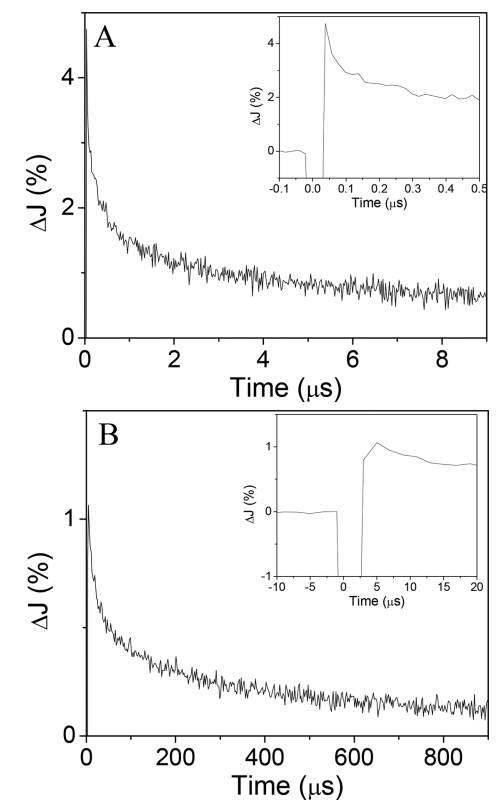

Figure 3. Change in reflectance $\Delta J$ of $\mathrm{TiO}_{2}$ at $500 \mathrm{~nm}-2$ ( 500 points) after laser excitation $\left(\lambda_{e x}=355 \mathrm{~nm}\right)$ observed for $10 \mu \mathrm{s}$ (A) and $1 \mathrm{~ms}$ (B). The insets show the signals at the very beginning of each measurement.

The inset of Figure 3 shows that the transient reflectance signal in the $10 \mu$ s measurement could be detected after $40 \mathrm{~ns}$. The initial negative peak is related to scattering of the UV laser (6 ns fwhm), which was used as excitation source. The terminal resistance of $50 \Omega$ facilitates a short rise time and allows the fast observation of the signal after $40 \mathrm{~ns}$. The high resistance of 5 $\mathrm{k} \Omega$ used for the $1 \mathrm{~ms}$ measurement leads to a much longer rise time of the detector. Accordingly, the first 5-6 $\mu$ s of the measurement do not represent the real transient signal and need to be removed before kinetic analysis. For all of the following calculations and figures, the charge carrier signals were analyzed after $40 \mathrm{~ns}$ for the $10 \mu \mathrm{s}$ measurement and after the first $6 \mu \mathrm{s}$ for the $1 \mathrm{~ms}$ measurement. The double logarithmic plot of the data in both time scales displays that the same signals could be detected after normalization (Figure 4).

In Figure $4 \mathrm{~B}$, the results are shown after the normalization. The normalization was carried out utilizing the overlapping region of 6-9 $\mu \mathrm{s}$, where the charge carrier signals should be identical. Because of the different experimental conditions, in particular the varying excitation energy and the terminal resistance, the signals display a different height. Subsequently, the signals were averaged in the overlapping time domain and the gained correction factor was multiplied with results from the $1 \mathrm{~ms}$ measurement. Hence, for the following kinetic analysis the normalization is generally considered. Double logarithmic plots with transient charge carrier signals observed in different time domains have already been used by Martin et al. for timeresolved microwave conductivity. ${ }^{11}$ The results achieved in this study correlate well with the results from Wang et al., who also 

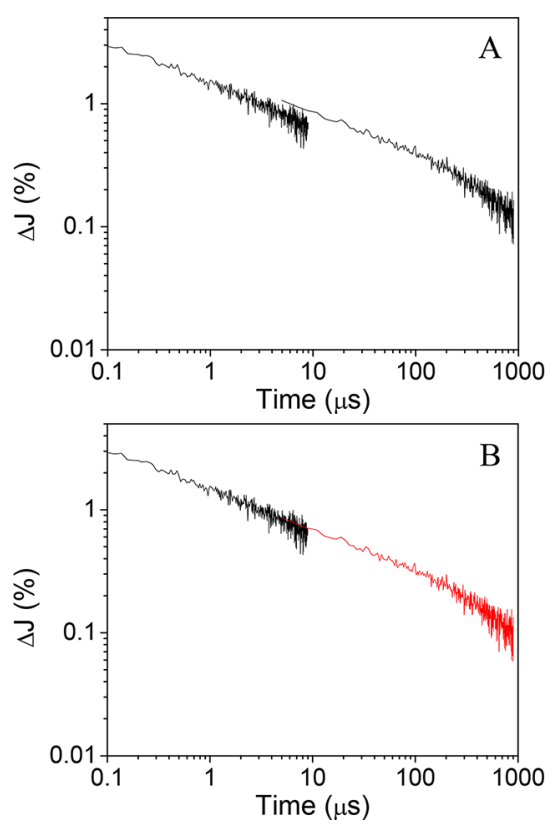

Figure 4. Change in reflectance $\Delta J$ of $\mathrm{TiO}_{2}$ at $500 \mathrm{~nm}-2\left(\lambda_{\text {ex }}=355\right.$ $\mathrm{nm}$ ) observed for the two time scales $10 \mu \mathrm{s}$ and $1 \mathrm{~ms}$ (A) and normalized signal (B).

received their results in two separated time domains $\left(10^{-5}\right.$ to 1 s). ${ }^{44}$

\section{RESULTS AND DISCUSSION}

Transient Reflectance Spectra. For all samples a broad transient reflectance between 400 and $700 \mathrm{~nm}$ was detected. This signal decays in the observed time domain from $50 \mathrm{~ns}$ until $1000 \mu \mathrm{s}$. Figure 5 displays the transient reflectance spectra at $0.1 \mu \mathrm{s}$ and at $7 \mu \mathrm{s}$ after the laser excitation.

Transient absorption peaks of trapped holes are typically observed at lower wavelength from 400 to $500 \mathrm{~nm}$ and peaks of trapped electrons have been identified at higher wavelength

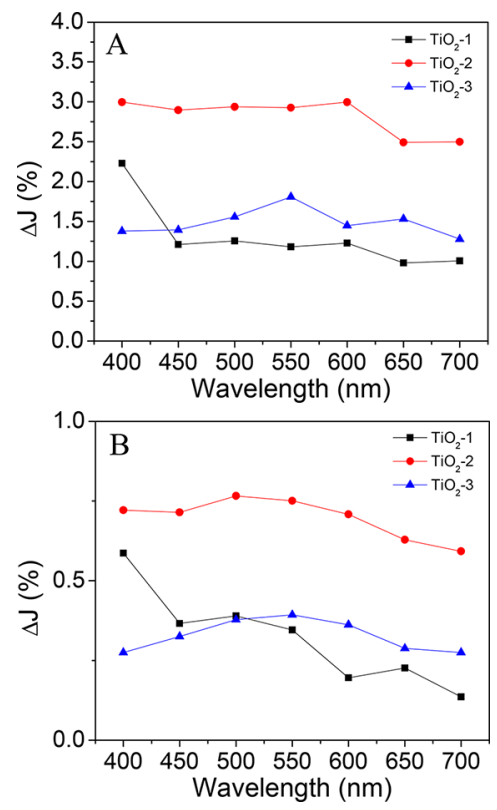

Figure 5. Transient reflectance spectra of the three $\mathrm{TiO}_{2}$ samples observed $0.1 \mu \mathrm{s}(\mathrm{A})$ and $7 \mu \mathrm{s}(\mathrm{B})$ after excitation $\left(\lambda_{\mathrm{ex}}=355 \mathrm{~nm}\right)$. between 600 to $800 \mathrm{~nm}{ }^{4}$ However, no apparent peak could be seen in the transient spectra between 400 and $700 \mathrm{~nm}$. This is in good agreement with the literature showing that the trapped charge carrier species absorb light over a broad range of wavelengths. ${ }^{17}$ In the experiments no reagent or scavenger has been employed and, subsequently, the transient reflectance signals represent a sum of trapped electron and trapped hole states. The absorption coefficients reported from Friedmann et al. correlate the transient absorption at $500 \mathrm{~nm}$ with a 50:50 mixture of trapped electrons and trapped holes. ${ }^{45}$ Nonetheless, the identification and separation of the charge carrier signals is not required. Since the experiment has been carried out under inert conditions, pure charge carrier recombination kinetics are observed. Direct comparison of the signals at $0.1 \mu \mathrm{s}$ (Figure $5 \mathrm{~A}$ ) and $7 \mu$ s (Figure 5B) indicates that the signals decay similar in the whole detected spectral domain. Hence, we used the traces at $500 \mathrm{~nm}$ as representatives for the kinetic analysis.

Second Order Decay Kinetics. Regularly, second order decay functions to fit the transient reflectance signals can be found in the literature. ${ }^{10}$ Assuming that both charge carriers only react with each other the two individual charge carrier concentrations can be replaced by one concentration. The concentration of the charge carriers $c$ is decaying from the initial concentration $c_{0}$ with the rate constant $k_{2}$ in eq 3 .

$$
\frac{1}{\mathrm{c}}=\frac{1}{c_{0}}+k_{2} t
$$

The variation of the concentration in course of the time can be shown by simple conversion to eqs 4 and 5 .

$$
\begin{aligned}
& \frac{1}{c}=\frac{1+c_{0} k_{2} t}{c_{0}} \\
& c=\frac{c_{0}}{1+c_{0} k_{2} t}
\end{aligned}
$$

To test the suitability of the second order fit function, eq 3 can be visualized by plotting the inverse signal $1 / c$ against the time $t$. For ideal second order reactions this plot should show a linear trend with the slope $k_{2}$. This kinetic analysis was done by Katoh et al. in 2008 for the transient absorption of a rutile single crystal in the time domain from 0 to $25 \mathrm{~ns}$ after excitation with $2.8 \mathrm{~mJ} / \mathrm{cm}^{2}{ }^{22}$ Figure 6 illustrates two of these second order kinetic plots for the sample of $\mathrm{TiO}_{2}-2$.

In Figure 6A, the charge carrier signal shows a nearly linear trend in the time domain 20 to $100 \mu \mathrm{s}$. Figure $6 \mathrm{~B}$ reveals that a linear trend can always be observed if the selected time domain is small enough. Overall, the charge carrier signal cannot be represented by a simple second order decay function. Nevertheless, the results confirm previously published results. Even for high excitation energies of up to $20 \mathrm{~mJ} / \mathrm{cm}^{2}$, charge carrier signals can successfully be fitted by second order decay functions if the observed or chosen time window is appropriately selected. ${ }^{10,42}$ The deviation from the ideal bimolecular behavior, which is visible especially for the borders of the selected time domain, is compensated by the addition of fitting parameters representing the residual signal. ${ }^{4,10,20}$ Equation 6 represents a typical second order fit function with baseline, following the scheme of eq 5 . To obtain eq 6, the extinction coefficient $\varepsilon$ was set to $1 \frac{\mathrm{L}}{\mathrm{mol} \cdot \mathrm{cm}}(A=\varepsilon c, A=c)$ and, as stated above, the linear relationship of the optical change in reflectance and the concentration of the transient species was also considered $(A \sim \Delta J){ }^{43,46}$ 

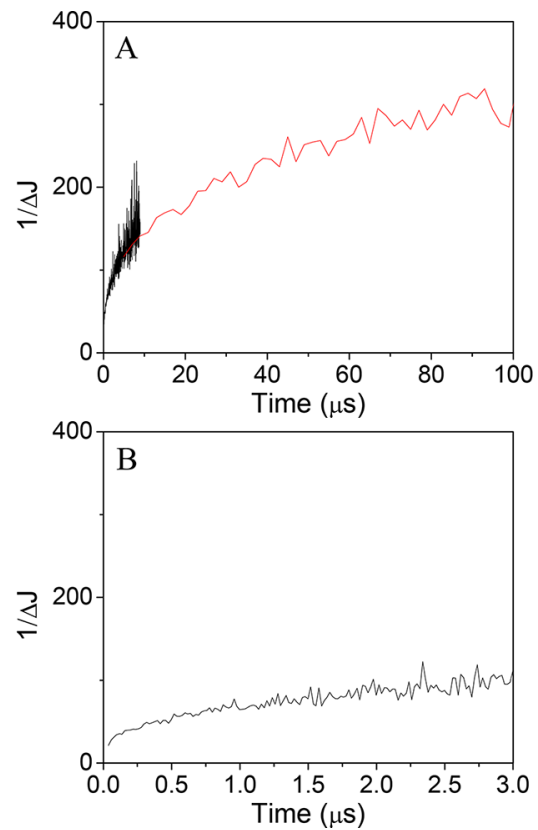

Figure 6. Reciprocal change in reflectance of $\mathrm{TiO}_{2}$ at $500 \mathrm{~nm}-2$ after excitation with $1 \mathrm{~mJ} / \mathrm{cm}^{2}$ in the time domains $100 \mu \mathrm{s}$ (A) and $3 \mu \mathrm{s}$ (B) taken from the $10 \mu \mathrm{s}$ measurement (black) and the $1 \mathrm{~ms}$ measurement (red).

$$
\Delta J=\frac{A}{1+A k_{2} t}+B
$$

The fitting parameter $A$ represents the height of the transient signal. $B$ is the residual signal at the end of the fit. The decay constant $k_{2}$ is related to the recombination constant of the photogenerated charge carriers. As described above the decay constant represents also the slope of the linear trend of the inverse signal $1 / c$ against the time $t$ (Figure 6). From the visual analysis of the data, it becomes obvious that the signal can be represented as a combination of linear functions with varied slope $k_{2}$. The slope, and accordingly the charge carrier recombination speed, is getting smaller in course of the time (Table 2). The full set of the fitting parameters is shown in the Supporting Information (Table S1).

Table 2. Results of Second Order Fit with Baseline (Eq 6) of the Change in Reflectance of $\mathrm{TiO}_{2}$ at $500 \mathrm{~nm}-2$ after Excitation with $1 \mathrm{~mJ} / \mathrm{cm}^{2}$

\begin{tabular}{lcccc} 
time window & $40 \mathrm{~ns}$ to $4.3 \mu \mathrm{s}$ & $4.3-9 \mu \mathrm{s}$ & $9-430 \mu \mathrm{s}$ & $430-899 \mu \mathrm{s}$ \\
$k_{2}(\mathrm{au})$ & 137.26 & 9.82 & 2.66 & 0.87 \\
\hline
\end{tabular}

The trends in Table 2 demonstrate that the second order decay constant is decreasing in course of the time. Therefore, the decay constant $k_{2}$ in eq 6 has been replaced by a time dependent term derived from fractal-like kinetics $\left(k_{2, f} t^{-h}\right)$ (cf. eq 7).

Fractal-Like Kinetics. The surface of one $\mathrm{TiO}_{2}$ particle can be described as nanostructured, possessing catalytic islands, irregular distributed trapping sites and relatively large variations in topography. ${ }^{47}$ Additionally to the fractal surface other fractal dimensions are formed by agglomerates in particulate suspensions, if the charge carrier transfer between different $\mathrm{TiO}_{2}$ particles is possible (Figure 7). This beneficial charge transfer between $\mathrm{TiO}_{2}$ particles has been described by Wang et

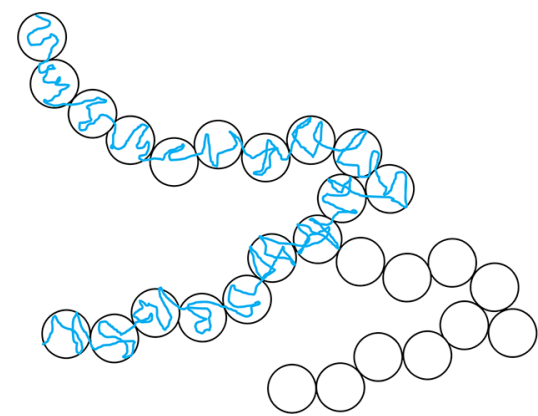

Figure 7. Schematic view of a fractal surface (black circles) and random pathway of a charge carrier (blue line). Adapted from ref 34.

al. in 2006 by the antenna mechanism. ${ }^{48}$ If we assume that the charge carriers are trapped close to the particle surface and the charges can freely move between the surfaces of the different particles, the giant agglomerated clusters of $\mathrm{TiO}_{2}$ particles represents a complex fractal.

Kopelman already described the use of time dependent kinetic constants for objects with fractal dimensions in 1988, which is in good agreement with the $t^{-1 / 2}$ dependence of the second-order rate coefficient identified by Grela and Colussi. $^{35,41}$ For batch reactions on fractal surfaces, where the diffusion is limited by the geometry, the rate constant $k_{2}$ needs to be multiplied by the time $t$ to the power of $-h$ (representing the rate coefficient $k_{f}$ ) eq $7 . .^{35}$

$$
k_{f}=k_{2, f} t^{-h} 0 \leq h \leq 1
$$

The exponent $\mathrm{h}$ represents the fractal dimension of the surface. In homogeneous media $(3 \mathrm{D})$ the exponent is equal to zero $(h=0)$ and classical time-independent kinetics are observed. However, for fractal surfaces the exponent differs from zero $h>0$ and fractal-like kinetics are observed. Typical values for $h$ of fractal surfaces are between $h=\frac{1}{3}$ and $h=\frac{1}{2} \cdot{ }^{35}$ Barzykin and Tachiya have shown that the mean-square displacement of a random walk on fractal structures displays an asymptotic $t^{a}$ behavior, where $a$ is the ratio between the spectral and the fractal dimensions. ${ }^{49}$ The asymptotic $t^{-1 / 2}$ dependence of the second-order rate coefficient, reported by Grela and Colussi in 1996, was first discussed by Toussaint and Wilczek for bimolecular reactions on square lattices at long times. $^{41,50}$ Additionally, the power-law model developed by Shuttle et al., ${ }^{27,28}$ which is used to represent the charge carrier recombination at long time scales and at low excitation energies, can be related to this asymptotic $t^{-1 / 2} \quad\left(h=\frac{1}{2}\right)$ dependence. Double logarithmic plots linearize eq 7 to eq 8 .

$$
\log \left(k_{2}\right)=\log \left(k_{2, f}\right)+(-h) \log (t), 0 \leq h \leq 1
$$

Subsequently, the slope of the double logarithmic plot of the rate constant against the time represents $h$ and is related to the fractal dimension of the system. For very long times $h$ equals 0.5 for $\mathrm{A}+\mathrm{B}$ reactions on square lattices. ${ }^{50}$

The mathematical description of the fractal-like kinetics from eq 7 was included in the second order fit function according to eq 5 and 6 , the result is shown in eq 9.

$$
\Delta J=\frac{A(1-h)}{(1-h)+A k_{2, f} t^{1-h}}
$$


Model of Sample Surface after Segregation Based on Second Order Kinetics. The agglomerated particles and the nanostructured surface of the $\mathrm{TiO}_{2}$ particles lead to a fractal dimension of the space available for the movement of the trapped charge carriers. Fractal surfaces are well-known to yield in segregation of the reactants. ${ }^{35}$ This segregation can easily be modeled by the sum of multiple surfaces following simple second order kinetics. Ten separated particles with different starting concentrations of charge carriers were selected. Such a disproportionate distribution of charge carriers can easily be created in the experiment without prior segregation if we assume that the absorption coefficient is not constant in the different particles of grains. All model parameters can be found in Table 3.

Table 3. Parameters of the Model of a Bimolecular Reaction on a Surface after Segregation

\begin{tabular}{ll} 
& \multicolumn{1}{c}{ values } \\
rate law & $-\frac{\mathrm{d}\left[\mathrm{e}^{-}\right]}{t}=k\left[\mathrm{e}^{-}\right]\left[\mathrm{h}^{+}\right]$ \\
$\begin{array}{l}\text { initial trapped charge carrier } \\
\text { concentrations }\end{array}$ & $\begin{array}{c}5000 ; 3500 ; 1000 ; 499 ; 50 ; 20 ; 10 ; 5 ; 2 ; \\
1 \text { charge carriers/particle }\end{array}$ \\
$\begin{array}{ll}\text { rate constant } & 10 \frac{\text { particle }}{\text { charge carriers } s} \\
\text { step size } & 1 \mu \mathrm{s} \\
\text { number of steps } & 2000\end{array}$ \\
\hline
\end{tabular}

The outcome of the model is illustrated in Figures 8 and 9, showing the concentration of the charge carriers in course of
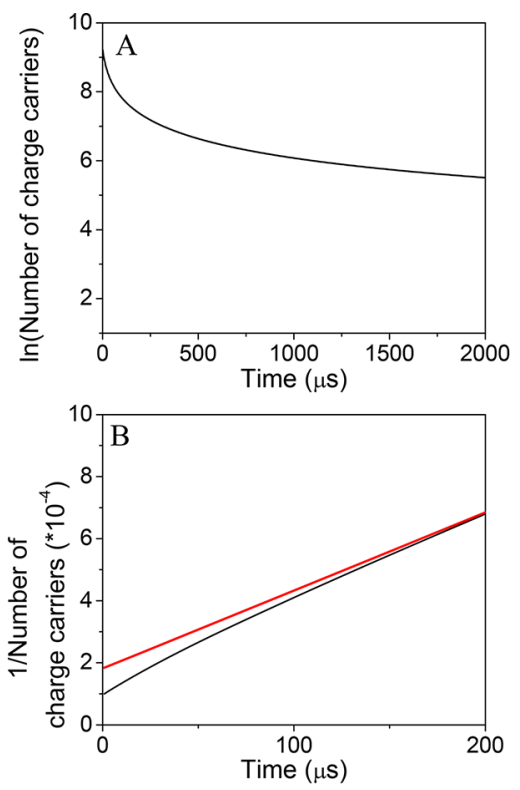

Figure 8. Logarithmic plot of the modeled charge carrier population (A) and inverse plot of the modeled charge carrier population (black) with linear fit (red) (B). The data points were taken from the model of a bimolecular reaction on the surface after segregation as defined in Table 3.

the time. As mentioned above each charge carrier concentration separately decays with second-order kinetics and the sum of the holes stay identical to the number of electrons. According to first order kinetics Figure 8A shows the logarithm of the charge carrier concentration obtained employing the model of a bimolecular reaction as a function of the elapsed time. If a

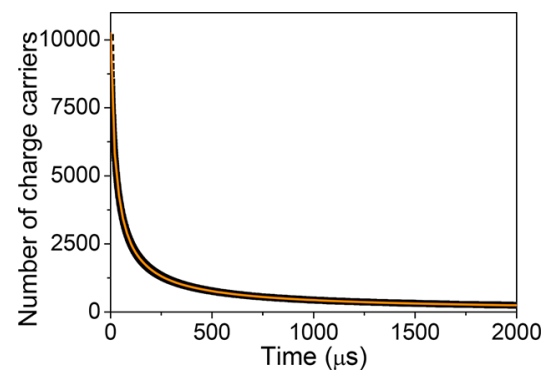

Figure 9. Course of the number of charge carriers taken from the model of a bimolecular reaction on the surface after segregation (black) and bimolecular fit based on fractal-like kinetics (orange).

reaction with first order kinetics would be observed, the logarithmic plot of the charge carrier concentration displays a linear behavior. No first order kinetic has been observed here for the modeled charge carrier concentration.

The inverse plot of the electron concentration (Figure 8B) reveals that similar to the experimental results shown in Figure 6 the modeled charge carrier concentration does not follow simple second-order kinetics. If the bimolecular reaction would display second-order kinetics the graph in Figure $8 \mathrm{~B}$ should easily be represented by a straight line. In particular, between 0 and $100 \mu \mathrm{s}$, the inverse charge carrier population derives from the second order behavior indicated by the red line. The observed number of charge carriers is smaller than expected for second order reactions. Accordingly, the simple model of the sum of ten surfaces with different charge carrier concentrations, each following simple second order kinetics, leads to a deviation of the second order kinetics which could successfully be fitted by the fractal-like kinetics approach (Figure 9).

The fitting parameters of the illustrated fractal-like kinetics can be found in Table 4. For easy comparison with the ideal second order behavior the parameters of the corresponding second order fit function are shown in Table 4 as well.

Table 4. Results of the Second Order Fit and the FractalLike Kinetics Fit $\left(\Delta J=\frac{A(1-h)}{(1-h)+A k_{2, f} t^{1-h}}\right)$ Applied on the Model of a Bimolecular Reaction on the Surface after Segregation

\begin{tabular}{|c|}
\hline fit function \\
\hline$k(\mathrm{au})$ \\
\hline$A(\mathrm{au})$ \\
\hline$h$ \\
\hline $\bar{R}^{2}$ \\
\hline
\end{tabular}

second order
2.68
9250
0
0.9918

fractal kinetics
0.73
10873
0.14
0.9999

A simple second order fit function displayed a much smaller correlation $\left(\bar{R}^{2}=0.992\right)$ with the modeled charge carrier signal than the fractal-like kinetics fit $\left(\bar{R}^{2}=0.9999\right)$. Because of the small number of modeled particles the deviation to the second order kinetics is small, as can be seen in Figure $8 \mathrm{~B}$. Subsequently, a small value was found for the fractal parameter $h$ (0.14). A higher number of different particles and a higher degree of complexity correspond to higher $h$ values.

Application of the Fractal Fit Function on the Experimental Data. Equation 9 was used to fit the observed charge carrier signals in $\mathrm{TiO}_{2}$ from $40 \mathrm{~ns}$ to $1 \mu \mathrm{s}$. The charge carrier signal and the fit function of $\mathrm{TiO}_{2}-2$ at $500 \mathrm{~nm}$ are displayed in Figure 10. 


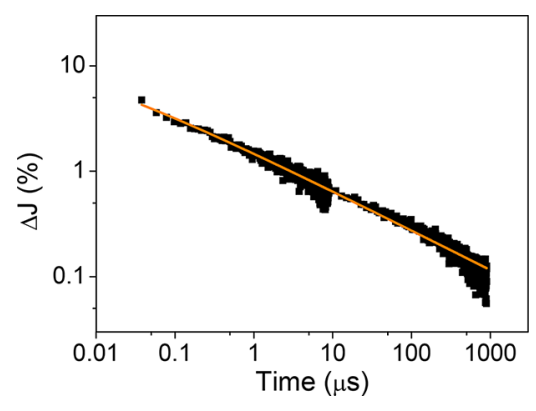

Figure 10. Optical change in reflectance $\Delta J$ at $500 \mathrm{~nm}$ of $\mathrm{TiO}_{2}-2$ (black) and bimolecular fit based on fractal-like kinetics (orange) in the time domain $40 \mathrm{~ns}$ to $1 \mathrm{~ms}$.

Very good agreement between the experimental results and the fitting curve were achieved without the need of an additional baseline $\left(\bar{R}^{2}=0.98\right)$. Hence, this model proves to be superior to a second order fit with an (arbitrary) baseline. In this study, the transient charge carrier signals were fitted with a parameter related to the fractal dimension of the semiconductor particles, instead of the addition of a baseline without any real physical meaning. To prove the applicability of the fractal kinetics approach the charge carrier signals of $\mathrm{TiO}_{2}-2$ were fitted at three different wavelengths, that is, 450, 500, and 550 $\mathrm{nm}$, with the obtained parameters these fits being shown in Table 5.

Table 5. Results of the Fractal Kinetics Fit of the Change in Reflectance at 450, 500, and $550 \mathrm{~nm}$ for $\mathrm{TiO}_{2}-2$ after Excitation with $1 \mathrm{~mJ} / \mathrm{cm}^{2}\left(\lambda_{e x}=355 \mathrm{~nm}\right)$ in the Time Domain from $40 \mathrm{~ns}$ to $1 \mathrm{~ms}$

\begin{tabular}{lccc} 
& wavelength $450 \mathrm{~nm}$ & wavelength $500 \mathrm{~nm}$ & wavelength $550 \mathrm{~nm}$ \\
$k_{2, f}(\mathrm{au})$ & 3.72 & 4.40 & 3.12 \\
$A(\mathrm{au})$ & 0.23 & 0.20 & 0.28 \\
$h$ & 0.64 & 0.62 & 0.64 \\
$\bar{R}^{2}$ & 0.9824 & 0.9826 & 0.9790 \\
\hline
\end{tabular}

In the spectroscopic range of 450-550 $\mathrm{nm}$, nearly the same kinetic parameters were observed, and the accuracy coefficients of their determination were close to 0.98 . This indicates that the same charge carrier signal is indeed observed at these selected wavelengths. As assumed from the transient reflectance spectra observed at different times after the laser excitation the broad charge carrier signal in the area of 400-600 nm decays with constant kinetic parameters. A rate coefficient of 3.1-4.4 has been identified and the fractal surface of the material causes the $t^{-h}$ dependence of the rate coefficient in course of the time. An exponent $h$ of $0.62-0.64$ has been observed for $\mathrm{TiO}_{2}-2$, which is close to the $t^{-1 / 2}$ dependence reported by Toussaint and Wilczek. ${ }^{50}$ Such a deviation can be expected for real complex powder surfaces, since the exponent is related to the structure of the fractal surface. The value of 0.5 is only observed for bimolecular reactions on square lattices and the fractal surface of the $\mathrm{TiO}_{2}-2$ powder, which most certainly deviates from a square even surface, results in an exponent of 0.6. To verify this influence of the fractal surface on the exponent $h$, charge carrier signals in $\mathrm{TiO}_{2}-1$ and $\mathrm{TiO}_{2}-3$ also have been investigated (Figure 11).

The displayed data in Table 5 and 6 reveal that for all three samples the average $h$ value range from 0.5 to 0.7 , which also correlates well with the asymptotic $\mathrm{t}^{-1 / 2}$ dependence.
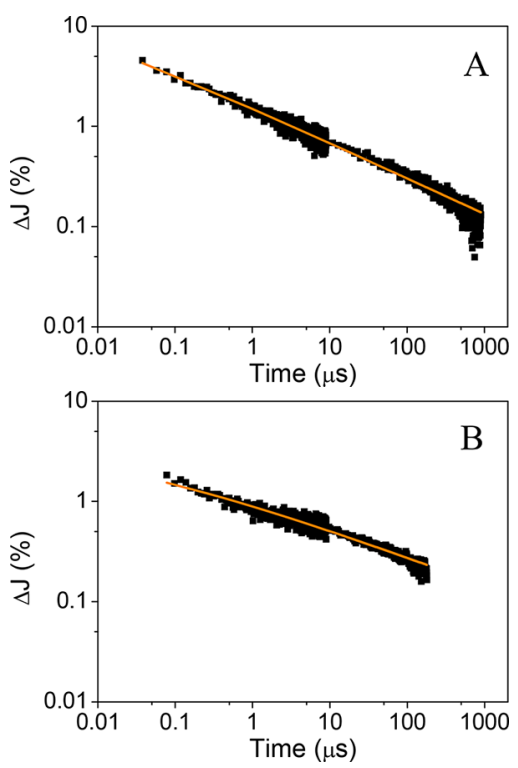

Figure 11. Change in reflectance $\Delta J$ at $500 \mathrm{~nm}\left(\lambda_{e x}=355 \mathrm{~nm}\right)$ (black) and bimolecular fit based on fractal-like kinetics (orange) of $\mathrm{TiO}_{2}-1$ (A) and $\mathrm{TiO}_{2}-3$ (B).

Table 6. Results of the Fractal Kinetics Fit of the Change in Reflectance at $500 \mathrm{~nm}$ for $\mathrm{TiO}_{2}-1$ and $\mathrm{TiO}_{2}-3$ after Excitation with $1 \mathrm{~mJ} / \mathrm{cm}^{2}\left(\lambda_{e x}=355 \mathrm{~nm}\right)$ in the Time Domain from $40 \mathrm{~ns}$ to $1 \mathrm{~ms}$

\begin{tabular}{lll} 
& \multicolumn{2}{c}{ sample } \\
\cline { 2 - 3 }$k_{2, f}(\mathrm{au})$ & $\mathrm{TiO}_{2}-1$ & $\mathrm{TiO}_{2}-3$ \\
$A(\mathrm{au})$ & 7.34 & 0.144 \\
$h$ & 0.020 & 0.046 \\
$\bar{R}^{2}$ & 0.47 & 0.71 \\
& 0.9177 & 0.9444
\end{tabular}

Nevertheless, the charge carrier signals in the three $\mathrm{TiO}_{2}$ powders display three different exponents $h$ proving the impact of the fractal surface on the parameter $h$ (Table 7 ).

Table 7. Exponent $h$ and Coefficient $k_{2, f}$ of the Fractal Kinetics Fit and BET Surface Area and Primary Particle Size of $\mathrm{TiO}_{2}-1, \mathrm{TiO}_{2}-2$, and $\mathrm{TiO}_{2}-3$

\begin{tabular}{lccl} 
& \multicolumn{3}{c}{ sample } \\
\cline { 2 - 4 } & $\mathrm{TiO}_{2}-1$ & $\mathrm{TiO}_{2}-2$ & $\mathrm{TiO}_{2}-3$ \\
$h(\mathrm{au})$ & 0.47 & 0.62 & 0.71 \\
$k_{2, f}(\mathrm{au})$ & 7.34 & 4.40 & 0.144 \\
$\mathrm{BET}\left(\mathrm{m}^{2} \cdot \mathrm{g}^{-1}\right)$ & 11 & 81 & 309 \\
primary particle aize $(\mathrm{nm})$ & 150 & 20 & 7.5 \\
\hline
\end{tabular}

The different particle sizes and particle size distributions lead to varying fractal surfaces in $\mathrm{TiO}_{2}-1, \mathrm{TiO}_{2}-2$, and $\mathrm{TiO}_{2}-3$. Table 7 indicates a trend of the parameter $h$ in course of the surface area of the samples. $\mathrm{TiO}_{2}-1$ has the largest particle size and displays an exponent of 0.47 in good agreement with the expected behavior on even surfaces. $\mathrm{TiO}_{2}-2$ and $\mathrm{TiO}_{2}-3$ show exponents of 0.62 and 0.71 , respectively, with the higher exponents being related with the smaller particle size of these samples. The small particles in $\mathrm{TiO}_{2}-2$ and $\mathrm{TiO}_{2}-3$ usually form agglomerates and this agglomeration changes the fractal surface from a relatively even surface of one particle to a complex 
three-dimensional surface of the agglomerate. Accordingly, the parameter $h$ changes from 0.47 for $\mathrm{TiO}_{2}-1$ to 0.7 for $\mathrm{TiO}_{2}-3$.

Border Cases and Comparison with the Power-Law Model. The literature utilizes second order decay functions for the initial part of the charge carrier signal and power-law decays at (very) long time scales. Both modes of data analysis can be explained as border cases of the fractal model described in the previous chapters. If the beginning of the decay of the charge carrier signal is investigated, for example in femtosecond studies, very short time scales are observed. Hence, the decay constant remains constant and simple second order kinetics can be observed via eq 10. The long lasting components of the signal which contain the complex fractal kinetics are usually masked by the arbitrary addition of a baseline (cf. eqs 3-5).

$$
k_{f} \approx k_{2, f} \times \text { const; } t^{-h} \approx \text { const, for } \Delta t \approx 0
$$

On the other hand, for the observation of charge carriers at long time scales after their formation the power-law model developed by Shuttle et al. has been applied. This model leads to a linear correlation between the height of the charge carrier signal and the elapsed time in a double logarithmic plot. ${ }^{27,28}$ The experimental results shown in Figures 4, 10, and 11 indicate a similar behavior. Nevertheless, Shuttle et al. already mentioned that the model is only applicable for small excitation energies and at long times. ${ }^{27}$ The measurements of the $\mathrm{TiO}_{2}$ powders shown in the figures mentioned above were performed under considerably low excitation energies of $1-2 \mathrm{~mJ} / \mathrm{cm}^{2}$. Hence, the experimental conditions for these measurements are close to the requirements for the model of Shuttle et al. and a linear dependency of the charge carrier signals upon the reaction time employing a double logarithmic presentation was to be expected. ${ }^{27}$ Subsequently, the power-law fit has been applied for the experiments carried out under higher excitation energy, that is $5 \mathrm{~mJ} / \mathrm{cm}^{2}$. Figure 12 displays the double
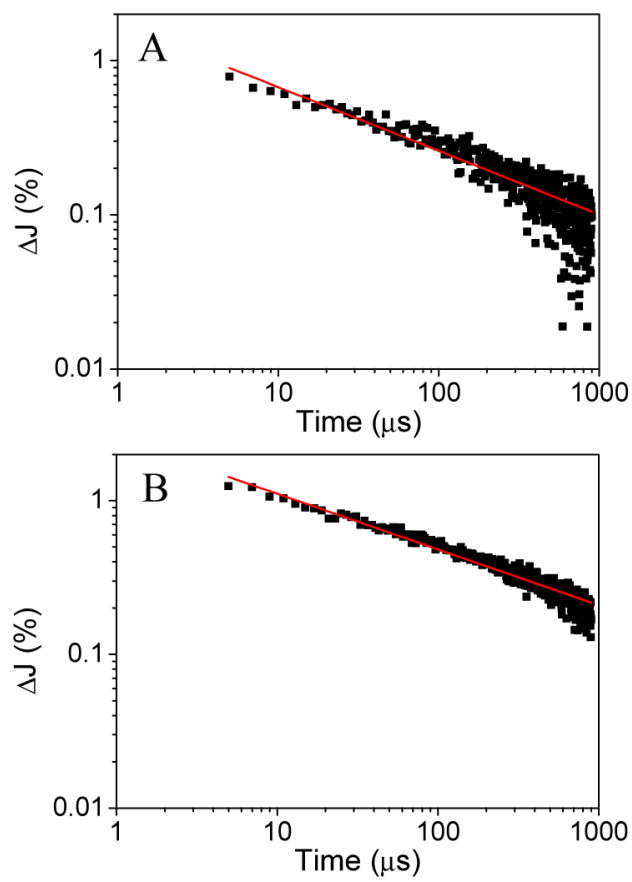

Figure 12. Double logarithmic plot of the optical change in reflectance of $\mathrm{TiO}_{2}-2$ at $500 \mathrm{~nm}$ after excitation with $0.6 \mathrm{~mJ} / \mathrm{cm}^{2}$ (A) and $5.1 \mathrm{~mJ} /$ $\mathrm{cm}^{2}$ (B) $\left(\lambda_{e x}=355 \mathrm{~nm}\right)$. logarithmic plots of the change in reflectance and the powerlaw fit functions $\left(\Delta J=A t^{-x}\right)$ at high and low excitation energies. The fitting parameters are listed in Table S2.

The power-law fitting process of the charge carrier signal with $5.1 \mathrm{~mJ} / \mathrm{cm}^{2}$ and with $0.6 \mathrm{~mJ} / \mathrm{cm}^{2}$ excitation energy results in exponents $x$ of 0.36 and 0.41 , respectively. Obviously, the exponent is larger for smaller excitation energy. This result indicates that charge carrier signals obtained employing high excitation energies such as $5.1 \mathrm{~mJ} / \mathrm{cm}^{2}$ can hardly be fitted with the power-law fit, since the slope of the linear fit function in the double logarithmic plot is changing during the course of the time (Figure 12). The asymptotic linear behavior of the charge carrier signals observed in the double logarithmic plot for low excitation energies and at long time scales taken from the power-law fit can be correlated with the fractal kinetics approach shown in eq 9. The double logarithmic plot leads to the following expression of the change in reflectance $\Delta J$ eq 11.

$$
\log (\Delta J)=\log (A(1-h))-\log \left((1-h)+A k_{2, f} t^{1-h}\right)
$$

For $t \gg 0, A k_{2 f} t^{1-h} \gg 1-h$; subsequently, $(1-h)+$ $A k_{2, f} t^{1-h} \approx A k_{2, f} t^{1-h}$. Therefore, eq 11 can be simplified to eq 12 , which reveals a linear dependence of the change in reflectance in course of the time.

$$
\log (\Delta J)=\log (A(1-h))-(1-h) \log \left(A k_{2, f} t\right),
$$$$
\text { for } t \gg 0
$$

Hence, the fractal kinetics fit results in an asymptotic linear behavior of the charge carrier signals in the double logarithmic plot similar to the power-law fit. Therefore, the power-law fit can be regarded as the border case for long times and at low excitation energies, with the slope in the power law $x$ being equal to $1-h^{27}$

The above-described model of a bimolecular reaction on a surface after segregation indicates the same linear trend in the double logarithmic plot (Figure 13) for long reaction times.

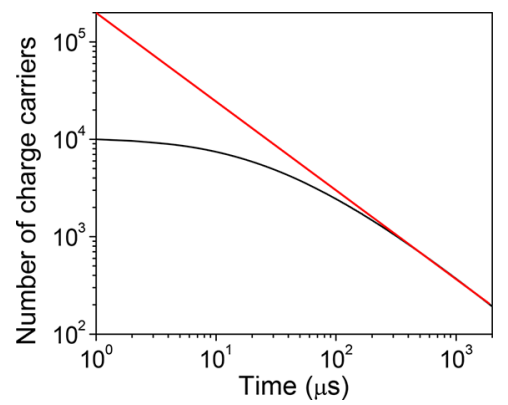

Figure 13. Double logarithmic plot of the electron concentration taken from the model of a bimolecular reaction on the surface after segregation (black) and straight line with slope -0.5 representing the asymptotic linear dependency (red).

Toussaint and Wilczek reported that at very long times the exponent $h$ equals to 0.5 for $\mathrm{A}+\mathrm{B}$ reactions on square lattices, thus also resulting in an exponent $x$ of 0.5 . $^{50}$

In good agreement with the results obtained at higher excitation energies, the model revealed that at the beginning the charge carrier signal does not follow a power-law trend. The slope in the double logarithmic plot is less negative at the beginning and approaches -0.5 at long times. This correlates 
well with the possible applications of the power-law model at long times and low excitation energies.

For excitation energies of $0.6 \mathrm{~mJ} / \mathrm{cm}^{2}$ an exponent $x$ of 0.41 has been observed for $\mathrm{TiO}_{2}-2$, which is close to the theoretical value of 0.5 for $\mathrm{A}+\mathrm{B}$ reactions on square lattices. Hence, for equal or smaller excitation energies and equal or longer time scales both models, the power-law model and the fractal kinetics approach, can be applied. However, for excitation energies exceeding $2 \mathrm{~mJ} / \mathrm{cm}^{2}$ and shorter time windows (40 ns to $1 \mathrm{~ms}$ ) the application of the fractal-like kinetics approach eq 9 is recommended.

\section{CONCLUSIONS}

In this study a kinetic model for the analysis of charge carrier kinetics based on fractal surfaces has been applied. The model successfully describes the charge carrier signal in the nanosecond to microsecond time scale observed in $\mathrm{TiO}_{2}$ following the excitation by a laser flash. Hence, it closes the gap between the well established second order processes in the short time scale (picoseconds to nanoseconds) and the power-law behavior in the long time scale (milliseconds to seconds). The reaction constant needs to be multiplied with $t^{-h}$, where the fractal parameter $h$ is related to the fractal structure of the material. Additionally, it was shown that the characteristic charge carrier signal can be simulated by a mathematical model based on segregation, a phenomenon strongly related to fractal surfaces. The mathematical model and the fractal-kinetics correlate well with the power-law model proposed by Shuttle et al., revealing the same asymptotic linear behavior in double logarithmic plots at long times and low excitation energies. ${ }^{27}$ Moreover, the fractal-kinetics approach enables the mathematical fitting of charge carrier signals in time scales ranging from nanoseconds to microseconds employing excitation energies of $1-5 \mathrm{~mJ} / \mathrm{cm}^{2}$.

\section{ASSOCIATED CONTENT}

\section{S Supporting Information}

The Supporting Information is available free of charge on the ACS Publications website at DOI: 10.1021/acs.jpcc.7b07087.

Table S1, results of the second order fit with baseline of the change in reflectance at $500 \mathrm{~nm}$ for $\mathrm{TiO}_{2}-2$ after excitation with $1 \mathrm{~mJ} / \mathrm{cm}^{2}\left(\lambda_{e x}=355 \mathrm{~nm}\right)$, and Table S2, results of the power-law fit of the change in reflectance at $500 \mathrm{~nm}$ for $\mathrm{TiO}_{2}-2$ after excitation with 0.6 and $5.1 \mathrm{~mJ} /$ $\mathrm{cm}^{2}\left(\lambda_{e x}=355 \mathrm{~nm}\right)(\mathrm{PDF})$

\section{AUTHOR INFORMATION}

\section{Corresponding Authors}

*(F.S.) E-mail: sieland@iftc.uni-hannover.de. Telephone +49 511762 16043. Webpage: https://www.tci.uni-hannover.de/. *(J.S.) E-mail: schneid@iftc.uni-hannover.de. Webpage: https://www.tci.uni-hannover.de/.

\section{ORCID $\odot$}

Fabian Sieland: 0000-0001-8640-6973

\section{Author Contributions}

All authors have given approval to the final version of the manuscript.

\section{Notes}

The authors declare no competing financial interest.

\section{ACKNOWLEDGMENTS}

Financial support from the Federal Ministry of Education and Research (BMBF) Project "PureBau" No. 13 N13350 is acknowledged. Moreover, we thank KRONOS International, Inc., and Crystal for the $\mathrm{TiO}_{2}$ powders. Additionally, F.S. acknowledges support from the Foundation of German Business (sdw).

\section{ABBREVIATIONS}

$\mathrm{TiO}_{2}$, titanium dioxide; $\mathrm{TiO}_{2}-3, \mathrm{PC} 500$ (Crystal); $\mathrm{TiO}_{2}-2$, PC105 (Crystal); $\mathrm{TiO}_{2}-1$, Kronos1001 (KRONOS International Inc.); $\mathrm{ABS}$, absorption; $\Delta J$, optical change in reflectance

\section{REFERENCES}

(1) Liu, B.; Zhao, X. A Kinetic Model for Evaluating the Dependence of the Quantum Yield of Nano- $\mathrm{TiO}_{2}$ Based Photocatalysis on Light Intensity, Grain Size, Carrier Lifetime, and Minority Carrier Diffusion Coefficient: Indirect Interfacial Charge Transfer. Electrochim. Acta 2010, 55 (12), 4062-4070.

(2) Reynal, A.; Lakadamyali, F.; Gross, M. a.; Reisner, E.; Durrant, J. R. Parameters Affecting Electron Transfer Dynamics from Semiconductors to Molecular Catalysts for the Photochemical Reduction of Protons. Energy Environ. Sci. 2013, 6 (11), 3291.

(3) Sze, S. M.; Ng, K. K.. Physics of Semiconductor Devices, 3rd. ed.; Wiley: 2007.

(4) Schneider, J.; Matsuoka, M.; Takeuchi, M.; Zhang, J.; Horiuchi, Y.; Anpo, M.; Bahnemann, D. W. Understanding $\mathrm{TiO}_{2}$ Photocatalysis: Mechanisms and Materials. Chem. Rev. 2014, 114, 9919-9986.

(5) Le Formal, F.; Pendlebury, S. R.; Cornuz, M.; Tilley, S. D.; Grätzel, M.; Durrant, J. R. Back Electron-Hole Recombination in Hematite Photoanodes for Water Splitting. J. Am. Chem. Soc. 2014, 136 (6), 2564-2574.

(6) Barroso, M.; Pendlebury, S. R.; Cowan, A. J.; Durrant, J. R. Charge Carrier Trapping, Recombination and Transfer in Hematite $\left(\alpha-\mathrm{Fe}_{2} \mathrm{O}_{3}\right)$ Water Splitting Photoanodes. Chem. Sci. 2013, 4, 2724.

(7) Serpone, N.; Lawless, D.; Khairutdinov, R. Size Effects on the Photophysical Properties of Colloidal Anatase $\mathrm{TiO}_{2}$ Particles: Size Quantization versus Direct Transitions in This Indirect Semiconductor? J. Phys. Chem. 1995, 99 (45), 16646-16654.

(8) Yan, J.; Wu, G.; Guan, N.; Li, L.; Li, Z.; Cao, X. Understanding the Effect of Surface/Bulk Defects on the Photocatalytic Activity of $\mathrm{TiO}_{2}$ : Anatase versus Rutile. Phys. Chem. Chem. Phys. 2013, 15 (26), 10978-10988.

(9) Bahnemann, D.; Henglein, A.; Lilie, J.; Spanhel, L. Flash Photolysis Observation of the Absorption Spectra of Trapped Positive Holes and Electrons in Colloidal Titanium Dioxide. J. Phys. Chem. 1984, 88 (4), 709-711.

(10) Furube, A.; Asahi, T.; Masuhara, H.; Yamashita, H.; Anpo, M. Charge Carrier Dynamics of Standard $\mathrm{TiO}_{2}$ Catalysts Revealed by Femtosecond Diffuse Reflectance Spectroscopy. J. Phys. Chem. B 1999, 103, 3120-3127.

(11) Martin, S. T.; Herrmann, H.; Choi, W.; Hoffmann, M. R. TimeResolved Microwave Conductivity. J. Chem. Soc., Faraday Trans. 1994 90 (21), 3315-3322.

(12) Tahiri Alaoui, O.; Herissan, A.; Le Quoc, C.; Zekri, M. E. M.; Sorgues, S.; Remita, H.; Colbeau-Justin, C. Elaboration, ChargeCarrier Lifetimes and Activity of $\mathrm{Pd}-\mathrm{TiO}_{2}$ Photocatalysts Obtained by Gamma Radiolysis. J. Photochem. Photobiol., A 2012, 242, 34-43.

(13) Katoh, R.; Tamaki, Y.; Furube, A. Primary Photocatalytic Reactions in $\mathrm{TiO}_{2}$ Nanoparticles Studied by Time-Resolved Laser Spectroscopy. Proc. SPIE 2011, 8109, 81090O-81090O-8.

(14) Murakami, Y.; Nishino, J.; Mesaki, T.; Nosaka, Y. Femtosecond Diffuse-Reflectance Spectroscopy of Various Commercially Available $\mathrm{TiO}_{2}$ Powders. Spectrosc. Lett. 2011, 44 (2), 88-94.

(15) Cowan, A. J.; Leng, W.; Barnes, P. R. F.; Klug, D. R.; Durrant, J. R. Charge Carrier Separation in Nanostructured $\mathrm{TiO}_{2}$ Photoelectrodes 
for Water Splitting. Phys. Chem. Chem. Phys. 2013, 15 (22), 87728778 .

(16) Bahnemann, D. W.; Hilgendorff, M.; Memming, R. Charge Carrier Dynamics at $\mathrm{TiO}_{2}$ Particles: Reactivity of Free and Trapped Holes. J. Phys. Chem. B 1997, 101 (21), 4265-4275.

(17) Yoshihara, T.; Katoh, R.; Furube, A.; Tamaki, Y.; Murai, M.; Hara, K.; Murata, S.; Arakawa, H.; et al. Identification of Reactive Species in Photoexcited Nanocrystalline $\mathrm{TiO}_{2}$ Films by WideWavelength-Range (400-2500 Nm) Transient Absorption Spectroscopy. J. Phys. Chem. B 2004, 108 (12), 3817-3823.

(18) Tamaki, Y.; Furube, A.; Katoh, R.; Murai, M.; Hara, K.; Arakawa, H.; Tachiya, M. Trapping Dynamics of Electrons and Holes in a Nanocrystalline $\mathrm{TiO}_{2}$ Film Revealed by Femtosecond visible/NearInfrared Transient Absorption Spectroscopy. C. R. Chim. 2006, 9 (2), 268-274.

(19) Draper, R. B.; Fox, M. A. Titanium Dioxide Photosensitized Reactions Studied by Diffuse Reflectance Flash Photolysis in Aqueous Suspensions of $\mathrm{TiO}_{2}$ Powder. Langmuir 1990, 6 (32), 1396-1402.

(20) Ohtani, B.; Bowman, R. M.; Colombo, D. P., Jr.; Kominami, H.; Noguchi, H.; Uosaki, K. Femtosecond Diffuse Reflectance Spectroscopy of Aqueous Titanium (IV) Oxide Suspension: Correlation of Electron-Hole Recombination Kinetics with Photocatalytic Activity. Chem. Lett. 1998, 27 (7), 579-580.

(21) Hoffmann, M. R.; Martin, S. T.; Choi, W.; Bahnemann, D. W. Environmental Applications of Semiconductor Photocatalysis. Chem. Rev. 1995, 95 (1), 69-96.

(22) Katoh, R.; Murai, M.; Furube, A. Electron-hole Recombination in the Bulk of a Rutile $\mathrm{TiO}_{2}$ Single Crystal Studied by SubNanosecond Transient Absorption Spectroscopy. Chem. Phys. Lett. 2008, 461 (4-6), 238-241.

(23) Schindler, K. M.; Kunst, M. Charge-Carrier Dynamics in Titania Powders. J. Phys. Chem. 1990, 94 (21), 8222-8226.

(24) Tachiya, M.; Mozumder, A. Kinetics of Geminate-Ion Recombination by Electron Tunneling. Chem. Phys. Lett. 1975, 34 (1), 77-79.

(25) Barzykin, A. V.; Tachiya, M. Mechanism of Charge Recombination in Dye-Sensitized Nanocrystalline Semiconductors: Random Flight Model. J. Phys. Chem. B 2002, 106 (17), 4356-4363.

(26) Nelson, J.; Haque, S.; Klug, D.; Durrant, J. Trap-Limited Recombination in Dye-Sensitized Nanocrystalline Metal Oxide Electrodes. Phys. Rev. B: Condens. Matter Mater. Phys. 2001, 63, 205321.

(27) Shuttle, C. G.; Hamilton, R.; Nelson, J.; O’Regan, B. C.; Durrant, J. R. Measurement of Charge-Density Dependence of Carrier Mobility in an Organic Semiconductor Blend. Adv. Funct. Mater. 2010, 20 (5), 698-702.

(28) Tang, J.; Durrant, J. R.; Klug, D. R. Mechanism of Photocatalytic Water Splitting in $\mathrm{TiO}_{2}$. Reaction of Water with Photoholes, Importance of Charge Carrier Dynamics, and Evidence for FourHole Chemistry. J. Am. Chem. Soc. 2008, 130 (42), 13885-13891.

(29) Pendlebury, S. R.; Barroso, M.; Cowan, A. J.; Sivula, K.; Tang, J.; Grätzel, M.; Klug, D.; Durrant, J. R. Dynamics of Photogenerated Holes in Nanocrystalline $\alpha$ - $\mathrm{Fe}_{2} \mathrm{O}_{3}$ Electrodes for Water Oxidation Probed by Transient Absorption Spectroscopy. Chem. Commun. (Cambridge, U. K.) 2011, 47 (2), 716-718.

(30) Wang, X.; Feng, Z.; Shi, J.; Jia, G.; Shen, S.; Zhou, J.; Li, C. Trap States and Carrier Dynamics of $\mathrm{TiO}_{2}$ Studied by Photoluminescence Spectroscopy under Weak Excitation Condition. Phys. Chem. Chem. Phys. 2010, 12 (26), 7083.

(31) Barzykin, A. V.; Tachiya, M. Stochastic Models of Charge Carrier Dynamics in Semiconducting Nanosystems. J. Phys.: Condens. Matter 2007, 19 (6), 065105.

(32) Mandelbrot, B. B. The Fractal Geometry of Nature. Am. J. Phys. 1983, 51, 286

(33) Avnir, D.; Farin, D.; Pfeifer, P. Chemistry in Noninteger Dimensions between Two and Three. I. Fractal Theory of Heterogeneous Surfaces. J. Chem. Phys. 1983, 79 (7), 3566.
(34) Le Mehaute, A.; Crepy, G. Introduction to Transfer and Motion in Fractal Media: The Geometry of Kinetics. Solid State Ionics 1983, 9-10, 17-30.

(35) Kopelman, R. Fractal Reaction Kinetics. Science (Washington, DC, U. S.) 1988, 241 (4873), 1620-1626.

(36) Hirakawa, T.; Nosaka, Y. Properties of $\mathrm{O}_{2}{ }^{-}$and $\mathrm{OH}^{\bullet}$ Formed in $\mathrm{TiO}_{2}$ Aqueous Suspensions by Photocatalytic Reaction and the Influence of $\mathrm{H}_{2} \mathrm{O}_{2}$ and Some Ions. Langmuir 2002, 18 (8), 32473254.

(37) Zhou, C.; Ma, Z.; Ren, Z.; Mao, X.; Dai, D.; Yang, X. Effect of Defects on Photocatalytic Dissociation of Methanol on $\mathrm{TiO}_{2}(110)$. Chem. Sci. 2011, 2 (10), 1980-1983.

(38) Feng, H.; Tan, S.; Tang, H.; Zheng, Q.; Shi, Y.; Cui, X.; Shao, X.; Zhao, A.; Zhao, J.; Wang, B. Temperature- and CoverageDependent Kinetics of Photocatalytic Reaction of Methanol on $\mathrm{TiO}_{2}(110)-(1 \times 1)$ Surface. J. Phys. Chem. C 2016, 120 (10), 55035514.

(39) Thompson, T. L.; Yates, J. T. Control of a Surface Photochemical Process by Fractal Electron Transport across the Surface: $\mathrm{O}_{2}$ Photodesorption from $\mathrm{TiO}_{2}(110)$. J. Phys. Chem. B 2006, 110 (14), 7431-7435.

(40) Niklasson, G. A. Fractal Aspects of the Dielectric Response of Charge Carriers in Disordered Materials. J. Appl. Phys. 1987, 62 (7), R1.

(41) Grela, M. A.; Colussi, A. J. Kinetics of Stochastic Charge Transfer and Recombination Events in Semiconductor Colloids. Relevance to Photocatalysis Efficiency. J. Phys. Chem. 1996, 100 (46), 18214-18221.

(42) Schneider, J.; Nikitin, K.; Wark, M.; Bahnemann, D. W.; Marschall, R. Improved Charge Carrier Separation in Barium Tantalate Composites Investigated by Laser Flash Photolysis. Phys. Chem. Chem. Phys. 2016, 18, 10719-10726.

(43) Lin, T.-P.; Kan, K. A. Calculation of Reflectance of a Light Diffuser with Nonuniform Absorption. J. Opt. Soc. Am. 1970, 60 (9), 1252.

(44) Wang, X.; Kafizas, A.; Li, X.; Moniz, S. J. A.; Reardon, P. J. T.; Tang, J.; Parkin, I. P.; Durrant, J. R. Transient Absorption Spectroscopy of Anatase and Rutile: The Impact of Morphology and Phase on Photocatalytic Activity. J. Phys. Chem. C 2015, 119 (19), 10439-10447.

(45) Friedmann, D.; Hansing, H.; Bahnemann, D. Primary Processes During the Photodeposition of Ag Clusters on $\mathrm{TiO}_{2}$ Nanoparticles. $Z$. Phys. Chem. 2007, 221 (3), 329-348.

(46) Kessler, R. W.; Krabichler, G.; Uhl, S.; Oelkrug, D.; Hagan, W. P.; Hyslop, J.; Wilkinson, F. Transient Decay Following Pulse Excitation of Diffuse Scattering Samples. Opt. Acta 1983, 30 (8), $1099-1111$.

(47) Arabatzis, I. M.; Antonaraki, S.; Stergiopoulos, T.; Hiskia, A.; Papaconstantinou, E.; Bernard, M. C.; Falaras, P. Preparation, Characterization and Photocatalytic Activity of Nanocrystalline Thin Film $\mathrm{TiO}_{2}$ Catalysts towards 3,5-Dichlorophenol Degradation. J. Photochem. Photobiol., A 2002, 149, 237-245.

(48) Wang, C.; Pagel, R.; Dohrmann, J. K.; Bahnemann, D. W. Antenna Mechanism and Deaggregation Concept: Novel Mechanistic Principles for Photocatalysis. C. R. Chim. 2006, 9 (5-6), 761-773.

(49) Barzykin, A. V.; Tachiya, M. Diffusion-Influenced Reaction Kinetics on Fractal Structures. J. Chem. Phys. 1993, 99 (12), 9591.

(50) Toussaint, D.; Wilczek, F. Particle-Antiparticle Annihilation in Diffusive Motion. J. Chem. Phys. 1983, 78 (5), 2642. 